\title{
Rohstoffpreise: Superzyklus oder Aufschwung?
}

In den vergangenen Monaten haben die Weltmarktpreise für Rohstoffe insbesondere für Industrierohstoffe stark zugelegt. Nach dem Einbruch im April 2020, ausgelöst durch den ersten globalen Lockdown zur Bekämpfung der Corona-Pandemie, sind die Rohstoffpreise kontinuierlich und zum Teil deutlich über das Vorkrisenniveau gestiegen (vgl. Abbildung 1). Auch die zwischenzeitlich erneut gestiegenen Corona-Infektionszahlen und die Einführung weiterer Lockdown-Maßnahmen konnten den Aufwärtstrend der Rohstoffpreise nicht bremsen. Der HWWI-Rohstoffpreisindex stieg im Vergleich zu seinem Vorkrisenniveau vom Dezember 2019 um $32 \%$. Wobei der Teilindex für Energierohstoffpreise einen Zuwachs von $28 \%$ und der Teilindex für Agrarrohstoffe einen Anstieg von $36 \%$ verzeichnete. Besonders beeindruckend ist jedoch der Anstieg des Teilindex für Industrierohstoffe, der gegenüber seinem Vorkrisenniveau um mehr als $70 \%$ zulegte und im Mai 2021 sein Zehnjahreshoch überschritt. Die Preiserhöhungen und Lieferengpässe waren für die Verbraucher:innen in den letzten Monaten vor allem bei Baumaterialien deutlich spürbar. In der öffentlichen Debatte wird daher bereits die Frage gestellt, ob diese enormen Preissteigerungen allein eine Auswirkung der Corona-Pandemie und damit von kurzer Dauer sind, oder den Beginn eines neuen Rohstoff-Superzyklus einläuten.

Rohstoffpreise sind sehr volatil und können durch kurzfristige angebots- oder nachfrageseitige Schocks stark beeinflusst werden. So sind die Preise für Agrarrohstoffe in hohem Maße von den Wetterbedingungen in den Anbauregionen abhängig. Die Preise für Industrierohstoffe werden in erster Linie nachfrageseitig von der weltweiten Industrieproduktion beeinflusst, jedoch können auch hier plötzliche Angebotsverknappungen, wie das schwere Ei-

\section{Abbildung 1}

Starker Anstieg der HWWI-Rohstoffpreisindizes

Tägliche Indexwerte auf US-\$-Basis, 2015=100

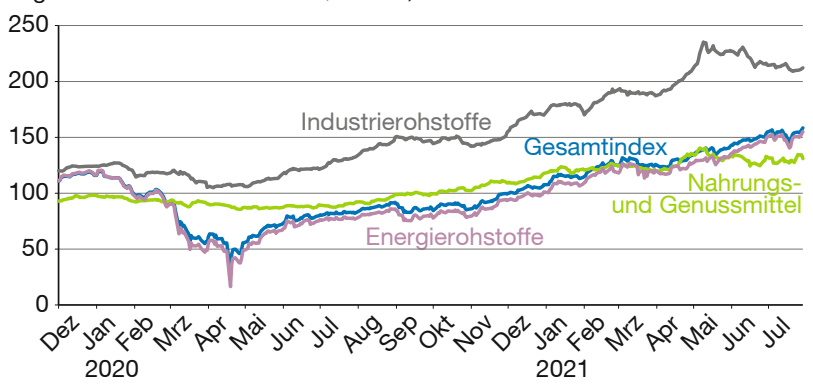

Quelle: Hamburgisches WeltWirtschaftsInstitut (HWWI). senerzminenunglück des Bergbauunternehmens Vale Anfang 2019 gezeigt hat, zu starken Preissteigerungen führen. Die Energierohstoffpreise werden zusätzlich durch geopolitische Konflikte oder durch die Politik der Organisation Erdöl exportierender Staaten (OPEC) beeinflusst. Trotz unterschiedlicher Treiber verlaufen die Rohstoffpreise über die verschiedenen Segmente hinweg nicht unabhängig voneinander. Empirische Studien wie die von Delle Chiaie et al. (2017) zeigen, dass die verschiedenen Rohstoffe durch einen gemeinsamen Faktor, der größtenteils durch die konjunkturelle Entwicklung der Weltwirtschaft erklärt wird, beeinflusst werden. So gehen Phasen des Aufschwungs der Weltwirtschaft mit einer erhöhten Nachfrage und Phasen des Abschwungs mit einem Rückgang der Nachfrage nach Rohstoffen einher (Schmidt et al., 2021). Dies zeigte sich deutlich, während der durch die Finanzkrise 2008/2009 ausgelösten globalen Rezession, die zu einem starken Einbruch der Rohstoffpreise in allen Rohstoffsegmenten führte (vgl. Abbildung 2).

Langfristige Trends der Rohstoffpreisentwicklung, die über die marktspezifischen Schwankungen und die konjunkturellen Zyklen hinausgehen, werden als Superzyklen bezeichnet. Ein Rohstoff-Superzyklus beschreibt eine lange Phase von nachfragegetriebenen Preissteigerungen, die deutlich über das durchschnittliche langfristige Preisniveau hinausgehen. Die Aufschwungsphase eines Superzyklus erstreckt sich über zehn bis 35 Jahre, da ein Strukturwandel der Wirtschaft die Nachfrage nach Rohstoffen nachhaltig verändert. Diese strukturelle Veränderung der Nachfrage erfordert eine strukturelle Angebotsanpassung, die je nach Rohstoff einige Zeit in Anspruch nehmen kann. Solange das Angebot aufgrund fehlender Kapazitäten nicht ausgeweitet werden kann, sorgt die steigende Nachfrage für Preisanstiege. In der Entwicklung der Rohstoffpreise während des vergangenen Jahrhunderts gab es mindestens drei solcher Superzyklen. So bewirkte z.B. die rasche Industrialisierung der USA in den späten 1800er bis in die frühen 1900er Jahre einen Rohstoff-Superzyklus. Der nächste Superzyklus wurde durch den Wiederaufbau Europas und den wirtschaftlichen Aufschwung Japans in der Nachkriegszeit ab 1945 ausgelöst. Der letzte Rohstoff-Superzyklus ab 2000 war auf die Industrialisierung und Urbanisierung der BRICLänder (Brasilien, Russland, Indien und China) und Chinas Beitritt zur Welthandelsorganisation zurückzuführen (vgl. Abbildung 2). Allen drei Superzyklen war gemeinsam, dass sie durch einen massiven Nachfrageschub ausgelöst wurden, der durch eine Transformation der Wirtschaft verur- 
Abbildung 2

Langfristige Entwicklung der HWWI-Rohstoffpreisindizes

Monatliche Indexwerte auf US-\$-Basis, 2015=100

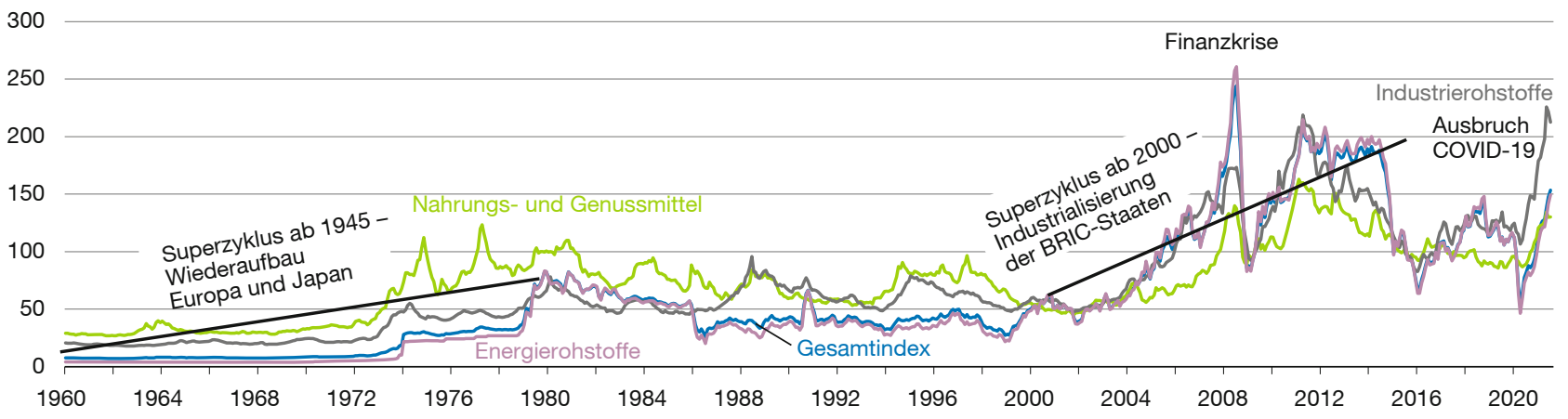

Quelle: Hamburgisches WeltWirtschaftsInstitut (HWWI).

sacht wurde und alle Rohstoffgruppen betraf (Heap, 2005; Cuddington und Jerrett, 2008; Erten und Ocampo, 2012).

Einigen Rohstoffanalyst:innen zufolge könnten die aktuellen Preissteigerungen im Zuge der wirtschaftlichen Erholung nach der Corona-Pandemie den Beginn eines neuen Superzyklus darstellen. In diesem Zusammenhang wird die COVID-19-Pandemie nicht als Ursache, sondern als Verstärkung einer bereits angelaufenen strukturellen Entwicklung gesehen. Die Digitalisierung und insbesondere die angestrebte Dekarbonisierung der Wirtschaft zur Erreichung der weltweit auferlegten Klimaziele bewirken einen technologischen Wandel, der einen strukturellen Nachfrageanstieg nach Rohstoffen mit sich ziehen könnte. Die globalen Lockdown-Maßnahmen haben die Digitalisierung durch die weltweite Zunahme an Remote-Arbeit und Homeschooling weiter beschleunigt. Die Dekarbonisierung wird derzeit zusätzlich durch die umfangreichen Konjunkturpakete unterstützt, die insbesondere auf klimafreundliche Technologien setzen. Bislang sind jedoch hauptsächlich bestimmte Rohstoffgruppen hiervon betroffen. So werden z.B. für Elektromobilität speziell Industriemetalle wie Kupfer, Nickel, Aluminium und Kobalt nachgefragt. Klimafreundliche Bauprojekte könnten hingegen den Verbrauch an Holz als nachhaltigem Baustoff steigern.

Bei dem rasanten Anstieg der Rohstoffpreise seit den Preiseinbrüchen im Frühjahr 2020 könnte es sich jedoch auch lediglich um einen kurzfristigen Aufschwung handeln. Die COVID-19-Pandemie hat die Rohstoffpreise über verschiedene Kanäle beeinflusst. Im Gegensatz zu früheren Rezessionen lösten die globale COVID-19-Pandemie und die Eindämmungsmaßnahmen eine einmalige Kombination von Angebots- und Nachfrageschocks auf den Rohstoffmärkten aus. Insbesondere auf den Märkten für Industrierohstoffe führten die Lockdown-Maßnahmen zu Angebotsverknappungen, da z.B. Minen temporär geschlossen und Lieferketten unterbrochen wurden. Da- rüber hinaus hat die rasche Erholung der chinesischen Wirtschaft die Nachfrage nach Industrierohstoffen bereits frühzeitig deutlich gesteigert. Die Nachfrage nach Baumaterialien für Renovierungsprojekte wurde zudem bereits während der Lockdown-Maßnahmen insbesondere in den USA durch den Privatsektor angeregt. Corona-Soforthilfen und umfangreiche Konjunkturpakete verstärkten den Nachfrageanstieg zusätzlich. Der gestiegenen globalen Nachfrage stand ein weiterhin geschwächtes Angebot gegenüber, was zu den enormen Preissteigerungen führte.

Die seit Mai wieder fallenden Preise an einigen Rohstoffmärkten deuten darauf hin, dass sich die Versorgungsengpässe allmählich auflösen. Der durch die Digitalisierung und die angestrebte Dekarbonisierung der Wirtschaft eingeleitete Strukturwandel wurde durch die Corona-Pandemie zwar unterstützt, treibt aber derzeit hauptsächlich die Nachfrage nach einem kleinen Teil der Industriemetalle wie z.B. Kupfer, Kobalt und Nickel. Ob diese Entwicklungen ausreichen, um einen neuen Superzyklus anzustoßen, ist daher derzeit noch fraglich.

Claudia Wellenreuther wellenreuther@hwwi.org

\section{Literatur}

Cuddington, J. und D. Jerrett (2008), Super Cycles in Real Metals Prices?, IMF Staff Paper, 55(4), 541-565.

Delle Chiaie, S., L. Ferrara und D. Giannone (2017), Common factors of commodity prices, ECB Working Paper, 2112.

Erten, B. und J. A. Ocampo (2012), Super-Cycles of Commodity Prices Since the Mid-Nineteenth Century, DESA Working Paper, 110, Februar, ST/ESA/DWP/110.

Heap, A. (2005), China - The Engine of a Commodities Super Cycle, Citigroup Smith Barney.

Schmidt, T., F. Kirsch und M. Dirks (2021), Kurzfristige Perspektiven der Rohstoffpreisentwicklung, Gutachten im Auftrag des Ministeriums für Wirtschaft, Innovation, Digitalisierung und Energie des Landes Nordrhein-Westfalens, RWI - Leibniz-Institut für Wirtschaftsforschung, Juli. 